\title{
Eficiência na remoção de NAT, DBO e DQO utilizando reator aeróbio de leito fluidizado com circulação em tubos concêntricos associado ao decantador de coluna em uma produção intensiva de tilápia
}

\author{
Removal efficiency of TAN, BOD and COD by using aerobic \\ fluidized bed reactor with circulation in concentric tubes associated \\ to a column settler in intensive production of tilapia
}

Tsunao Matsumoto', Yemall Alexander Maigual Enriquez ${ }^{2}$

\begin{abstract}
RESUMO
O desenvolvimento da aquicultura intensiva é acompanhado de um crescente impacto ambiental produzido pelos efluentes gerados. Os sistemas de recirculação para aquicultura (SRA) são uma alternativa compacta e trabalham com elevadas densidades de estocagem e menores volumes de água. Foi utilizado um decantador de coluna (DC) associado ao reator aeróbio de leito fluidizado com circulação em tubos concêntricos (BAS-CT) com tempo de retenção hidráulica (TRH) de 0,55 e 0,20 h respectivamente, num SRA com tilápias de 0,32 kg de peso médio e densidade de estocagem de $33,1 \mathrm{~kg} \cdot \mathrm{m}^{3}$ em relação à remoção do nitrogênio amoniacal total (NAT), demanda bioquímica de oxigênio (DBO) e demanda química de oxigênio (DQO). As eficiências na remoção de NAT, DBO e DQO foram 35,2, 48,0 e 64,9\%, respectivamente. No uso de DC com o BAS-CT, obteve-se bom desempenho, com baixos TRH no tratamento do efluente.
\end{abstract}

Palavras-chave: decantador de coluna; reator aeróbio de leito fluidizado; sistemas de recirculação para aquicultura; tempo de retenção hidráulica; tratamento de águas residuárias.

\begin{abstract}
The development of intensive aquaculture is accompanied by an increasing environmental impact produced by the wastewater generated. The recirculating aquaculture systems (RAS) are a compact alternative and manage high stocking densities and lower volumes of water. It was used a column settler (CS) associated with aerobic fluidized bed reactor (BAS-CT) with hydraulic retention time (HRT) of 0.55 and 0.20 h respectively, in RAS with tilapias with average weight of $0.32 \mathrm{~kg}$ and storage density of $33.1 \mathrm{~kg} \cdot \mathrm{m}^{-3}$ regarding the removal of total ammonia nitrogen (TAN), biochemical oxygen demand (BOD) and chemical oxygen demand (COD). The removal efficiencies of TAN BOD and COD were 35.2, 48.0 and 64.9\%, respectively. The use of CS with the BAS-CT showed a good performance with low HRT in the treatment of this effluent.
\end{abstract}

Keywords: column settler; aerobic fluidized bed reactor; recirculating aquaculture systems; hydraulic retention time; wastewater treatment.

\section{INTRODUÇÃO}

A intensificação da aquicultura no mundo inteiro tem provocado um incremento na concentração de nutrientes nos corpos receptores, aumentando a matéria orgânica e inorgânica, o que resulta na presença de sólidos dissolvidos, processo conhecido como hipernitrificação dos corpos hídricos. Além disso, também promove a introdução de outros resíduos nocivos, como substâncias químicas e antibióticos, que podem poluir o meio ambiente trazendo consigo todo um novo conjunto de preocupações ambientais (VAN DEN HENDE et al., 2014; PIEDRAHITA, 2003). O incremento de nutrientes é estimado entre

'Doutor em Hidráulica e Saneamento pela Escola de Engenharia de São Carlos da Universidade de São Paulo (USP). Professor Adjunto II do Departamento de Engenharia Civil da Faculdade de Engenharia de Ilha Solteira, Universidade Estadual Paulista "Júlio de Mesquita Filho" (UNESP) - Ilha Solteira (SP), Brasil.

${ }^{2}$ Doutorando pelo Instituto de Ciência e Tecnologia da UNESP - Sorocaba (SP), Brasil.

Endereço para correspondência: Tsunao Matsumoto - Faculdade de Engenharia de Ilha Solteira, Departamento de Engenharia Civil, Alameda Bahia, 550 - Centro - 15385 -000 Ilha Solteira (SP), Brasil - E-mail: tsunao@dec.feis.unesp.br

Recebido: 07/03/12 - Aceito: 16/02/16 - Reg. ABES: 84178 
80 e $88 \%$ em carbono e de 52 a 95\% em nitrogênio, os quais são introduzidos pela ração fornecida para os peixes, que os transformam em matéria particulada, íons químicos dissolvidos ou gases (GUTIERREZWING \& MALONE, 2006).

Os sistemas de recirculação em aquicultura (SRA) são um meio alternativo de produção intensiva de peixes em regiões com limitada disponibilidade hídrica, oferecendo potencial de produção tanto em pequena como em grande escala por conta da recuperação da qualidade dos efluentes pelo tratamento e reutilização da água no sistema de produção. Utilizam baixos volumes de água em função da taxa de evaporação baixa, ao contrário dos sistemas tradicionais de cultivo. Além dessa perda, também há retirada de água contendo resíduos líquidos e sólidos antes que eles confiram condições tóxicas ao meio aquático, devendo essa água ser reposta (BURUT-ARCHANAI et al., 2013; MAIGUAL; SÁNCHEZ; MATSUMOTO, 2013; SCHREIER; MIRZOYAN; SAITO, 2010; PFEIFFER; OSBORN; DAVIS, 2008).

A presença e o acúmulo de cargas orgânicas e nitrogenadas originadas da ração não consumida e dos processos metabólicos próprios dos animais em cultivo tendem a diminuir a concentração de oxigênio dissolvido (OD), exercendo aumento na concentração da demanda bioquímica de oxigênio (DBO), demanda química de oxigênio (DQO), produção e mineralização de diferentes formas de nitrogênio, como o nitrogênio amoniacal, amônia não ionizada e nitritos, que são tóxicos para os peixes (DOLAN; MURPHY; O'HEHIR, 2013; MIRZOYAN; TAL; GROSS, 2010; JOHNSON \& CHEN, 2006; LAWSON, 1995).

Para Gutierrez-Wing e Malone (2006), atualmente a remoção da maior parte dos poluentes nos SRA é feita por intermédio de sistemas de tratamento biológico, entre os quais estão os reatores de leito fluidizados tipo biofilm airlift suspension (BAS), chamados também de loop reactors, que possuem duas seções conectadas: uma no tubo de subida, conhecido como riser, no qual o meio trifásico, composto por líquido, ar e meio de suporte (areia, carvão ativado, material plástico), circula em fluxo ascendente por jatos de ar; e a outra região no tubo de descida, chamada de downcomer, em fluxo do topo para a base, podendo ter a configuração de tubos concêntricos (CT) (LEKANG, 2013; NICOLELLA; VAN LOOSDRECHT; HEIJNEN, 2000).

Reatores tipo BAS são empregados em diferentes partes do mundo como parte dos SRA, removendo de 50 a 90\% do nitrogênio amoniacal total (NAT) em cada passagem da água pelo sistema e mantendo, dessa forma, as concentrações baixas no cultivo. Esses sistemas podem tratar vazões de água da ordem de $0,19 \mathrm{~m}^{3} \cdot \mathrm{s}^{-1}$, em razão da formação de biofilme em volta de pequenas partículas suspensas, como a areia ou o carvão granular, usadas como meio de suporte, além de possibilitar a compacidade dos reatores, disponibilidade de materiais e manutenção dos equipamentos (SÁNCHEZ ORTIZ et al., 2010; SUMMERFELT, 2006).

O objetivo do presente estudo foi determinar a eficiência do sistema de tratamento de águas residuárias em relação à remoção de NAT, DBO e DQO na produção intensiva de tilápias em sistema de recirculação.

\section{METODOLOGIA}

O trabalho foi realizado nas dependências dos laboratórios de Hidrologia e Hidrometria $\left(\mathrm{LH}^{2}\right)$ e de Saneamento da Faculdade de Engenharia do Campus de Ilha Solteira, da Universidade Estadual Paulista Júlio de Mesquita Filho (UNESP). As análises dos parâmetros físico-químicos do efluente gerado no SRA seguiram as recomendações do Standard methods for examination of water and wastewater (APHA, 2005).

O SRA era composto por caixa de nível constante, tubulações de policloreto de vinila (PVC) para distribuição de água aos tanques de cultivo e calha semicircular para o controle do nível e coleta do efluente dos tanques. O SRA ainda possuía um decantador de coluna (DC) para remoção de sólidos sedimentáveis, e na sequência foram instalados o reator aeróbio de leito fluidizado com circulação em tubos concêntricos, ou biofilm airlift suspension (BAS-CT), e o reator de remoção de gás carbônico. Após a passagem do afluente por esses dispositivos, o efluente tratado foi encaminhado ao tanque de sucção do sistema de recalque que conduzia a água ao tanque de nível constante.

Para o sistema de tratamento de efluentes foi construído um DC em PVC de $400 \mathrm{~mm}$ de diâmetro, 2,50 m de altura e volume de $0,30 \mathrm{~m}^{3}$. O tempo de retenção hidráulica (TRH) no DC foi de 0,55 h para remoção de sólidos maiores ou iguais a $0,2 \mathrm{~mm}$.

O BAS-CT foi construído em PVC com $250 \mathrm{~mm}$ de diâmetro e 2,50 m de altura no downcomer, e no riser com PVC de $100 \mathrm{~mm}$ de diâmetro e 2,20 m de altura. O TRH do reator foi de $0,20 \mathrm{~h}$, usando como meio de suporte areia para pré-filtro com $\mathrm{d}_{10}=0,16 \mathrm{~mm}$ numa concentração de 80 g.L $\mathrm{L}^{-1}$. A vazão de ar injetado na base BAS-CT para fluidizar a areia foi de $2.500{\mathrm{~L} . \mathrm{h}^{-1}}^{-1}$ no início do projeto e, após o crescimento

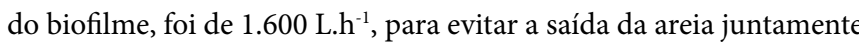
com as bactérias encarregadas de realizar o tratamento do efluente.

Também foi instalado um reator para transferência de oxigênio e remoção de $\mathrm{CO}_{2}$ em PVC de $300 \mathrm{~mm}$ de diâmetro e $1,80 \mathrm{~m}$ de altura e TRH de 0,22 h. No sistema de geração de ar pressurizado se utilizaram um compressor de $7,5 \mathrm{Cv}$, mangueiras plásticas para distribuição e injeção, um tanque de sucção de $0,25 \mathrm{~m}^{3}$, uma bomba centrífuga de $1 / 2 \mathrm{Cv}$ no sistema de recalque do efluente tratado e um soprador de ar de 1,0 Cv, empregado para eliminação do cloro na água de troca.

Os pontos de coleta de amostras para a avaliação do sistema de tratamento de efluentes no SRA foram os seguintes: entrada do DC (E.DC), saída do DC (S.DC) e saída do BAS-CT (S.BAS-CT). No presente estudo foram monitorados os seguintes parâmetros de controle de tratamento de águas residuárias: NAT, DBO e DQO no sistema fechado do tipo SRA. 
O efluente foi gerado nos três tanques de cultivo de tilápia-do-nilo do SRA com densidade de estocagem inicial aproximada de $17 \mathrm{~kg} \cdot \mathrm{m}^{-3} \mathrm{e}$ vazão de água de entrada de $0,0097 \mathrm{~m}^{3} \cdot \mathrm{s}^{-1}$, distribuída nos três tanques para criação de peixes. Cada tanque possuía volume útil de $0,20 \mathrm{~m}^{3}$, e a alimentação dos peixes foi realizada com ração comercial, com conteúdo de $40 \%$ de proteína à razão de $1,2 \%$ da biomassa de peixe por dia.

Para a limpeza do DC, optou-se por três descargas ao dia, com volume aproximado de $0,03 \mathrm{~m}^{3}$ por descarga. Esse procedimento representou renovação diária de 6,1\% do volume total de água do SRA.

A água de reposição do SRA era proveniente da rede de abastecimento, a qual foi previamente desclorada num tanque de armazenamento aerado para essa finalidade. A água de reposição do SRA era adicionada ao tanque de sucção do sistema. A Figura 1 apresenta o esquema geral do SRA utilizado neste estudo.

\section{RESULTADOS E DISCUSSÃO}

Na Tabela 1 são apresentados os resultados das concentrações máxima, mínima e média para os três parâmetros avaliados ao longo deste estudo.

Durante o período de ensaios, notou-se acúmulo de sólidos na zona da saída dos tanques de cultivo, na qual esses sólidos começavam a flutuar, liberando bolhas de gás, provavelmente produzidas pela decomposição anaeróbia da matéria orgânica ou ração não consumida, gerando gás sulfídrico $\left(\mathrm{H}_{2} \mathrm{~S}\right)$, metano $\left(\mathrm{CH}_{4}\right)$ e outros gases odoríferos que poderiam ter influenciado na aparição eventual de concentrações elevadas dos parâmetros avaliados (MIRZOYAN; TAL; GROSS, 2010).

\section{Remoção do nitrogênio amoniacal total (NAT)}

Em todo SRA o biorreator é comumente utilizado para manter os níveis de NAT em concentrações não letais aos peixes, considerando a faixa de segurança para a sobrevivência entre 0,1 e $3 \mathrm{mg} \cdot \mathrm{L}^{-1}$, e a amônia não ionizada $\left(\mathrm{NH}_{3}-\mathrm{N}\right)$ deve ser mantida em concentrações $<0,6$ mg.L $\mathrm{L}^{-1}$ (SÁNCHEZ ORTIZ \& MATSUMOTO, 2012; SCHREIER; MIRZOYAN; SAITO, 2010). A média calculada na S.BAS-CT ficou abaixo desses limites (Tabela 1), mostrando que o sistema foi eficiente na oxidação e remoção de NAT.

Tabela 1 - Concentrações máxima, mínima e média do nitrogênio amoniacal total, demanda bioquímica de oxigênio e demanda química de oxigênio no sistema de recirculação em aquicultura.

\begin{tabular}{l|c|c|c|c}
\multirow{3}{*}{ Parâmetro } & \multirow{2}{*}{ Ponto } & \multicolumn{3}{|c}{ Concentração (mg.L-1) } \\
\cline { 3 - 5 } & & Máxima & Mínima & Média \\
\cline { 2 - 5 } & E.DC & 4,58 & 0,11 & 1,12 \\
\cline { 2 - 5 } & S.DC & 3,55 & 0,06 & 0,99 \\
\cline { 2 - 5 } & S.BAT-CT & 2,98 & 0,03 & 0,81 \\
\hline \multirow{4}{*}{ DBO } & E.DC & 7,54 & 3,89 & 5,67 \\
\cline { 2 - 5 } & S.DC & 5,74 & 0,84 & 3,38 \\
\cline { 2 - 5 } & S.BAS-CT & 5,23 & 0,63 & 2,65 \\
\hline \multirow{3}{*}{ DQO } & E.DC & 83,00 & 5,00 & 40,48 \\
\cline { 2 - 5 } & S.DC & 34,00 & 4,00 & 13,96 \\
\cline { 2 - 5 } & S.BAS-CT & 22,00 & 1,00 & 10,09 \\
\hline
\end{tabular}

NAT: nitrogênio amoniacal total; DBO: demanda bioquímica de oxigênio; DQO: demanda química de oxigênio; E.DC: entrada do decantador de coluna; S.DC: saída do decantador de coluna; S.BAS-CT: saída do reator aeróbio de leito fluidizado com circulação em tubos concêntricos.

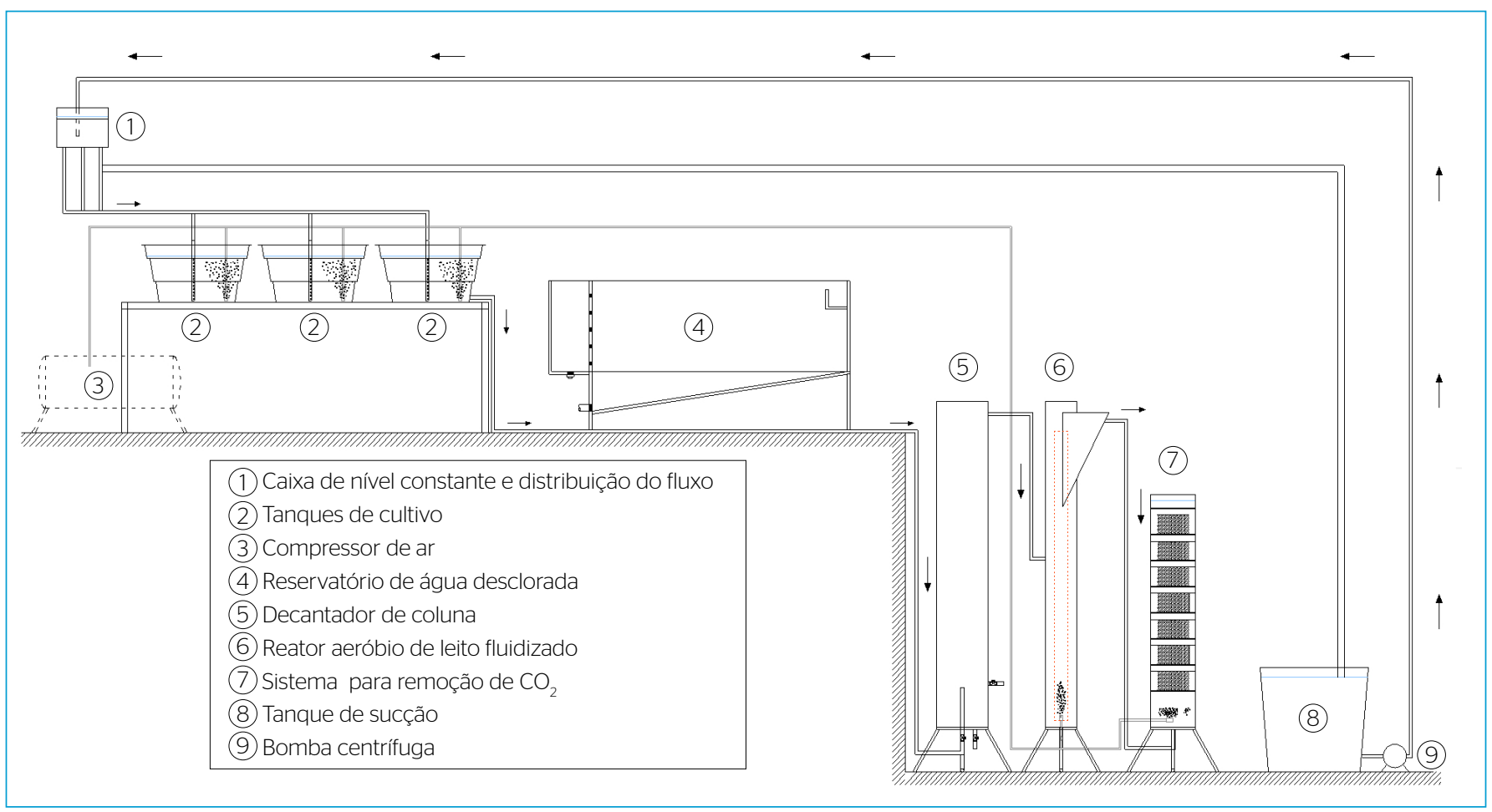

Figura 1 - Perfil dos componentes do sistema de recirculação para tilápias. 
Na Figura 2 são apresentados os valores de NAT nos diferentes pontos amostrados neste estudo. No caso de NAT, a eficiência de remoção foi de $35,2 \%$ para o período após a estabilização das concentrações de NAT no BAS-CT. Esse resultado também foi melhor se comparado com os resultados alcançados por Sánchez Ortiz e Matsumoto (2012), que obtiveram remoção de $27 \%$ no BAS-CT. Ainda, foram superiores aos reportados por Timmons et al., (2002) e Summerfelt (2006), com eficiências de remoção de NAT de 8 a 11\% para meio suporte de areia com $D_{10}$ entre 0,45 e 0,80 mm, utilizado no reator de leito fluidizado bifásico.

Para Brazil (2006), com um contador biológico rotativo, obteve-se redução de aproximadamente $40 \%$ de NAT do efluente de SRA com produção de tilápia. Davidson, Helwig e Summerfelt (2008), mediante um reator de leito fluidizado (FSB) com areia $\mathrm{D}_{10}=0,11 \mathrm{~mm}$, constataram que a remoção de NAT se manteve na faixa de 62 a $63 \%$. Com areia de $\mathrm{D}_{10}=0,19 \mathrm{~mm}$, a remoção foi de $30 \%$, sendo este um valor similar ao obtido na presente pesquisa, mas com areia $\mathrm{D}_{10}=0,16 \mathrm{~mm}$, demonstrando assim a influência da superfície específica do meio de suporte na capacidade de crescimento de biofilme, portanto, na eficiência na remoção de NAT das águas residuárias.

\section{Remoção da demanda bioquímica de oxigênio (DBO)}

A concentração da DBO na E.DC sempre foi maior do que na S.DC e na S.BAS-CT. A diminuição desses resultados pode ser interpretada como adequada, visando dessa forma à melhoria da qualidade da água quando esta volta para os tanques de cultivo. Alguns picos de DBO observados (Tabela 1) podem estar ligados a erros no procedimento da metodologia utilizada, mas também ser atribuídos ao acúmulo de matéria orgânica acumulada no sistema de drenagem dos tanques e à ração não consumida. Possivelmente, o acúmulo de sólidos no reator foi em razão do crescimento do biofilme, resultando em acréscimo

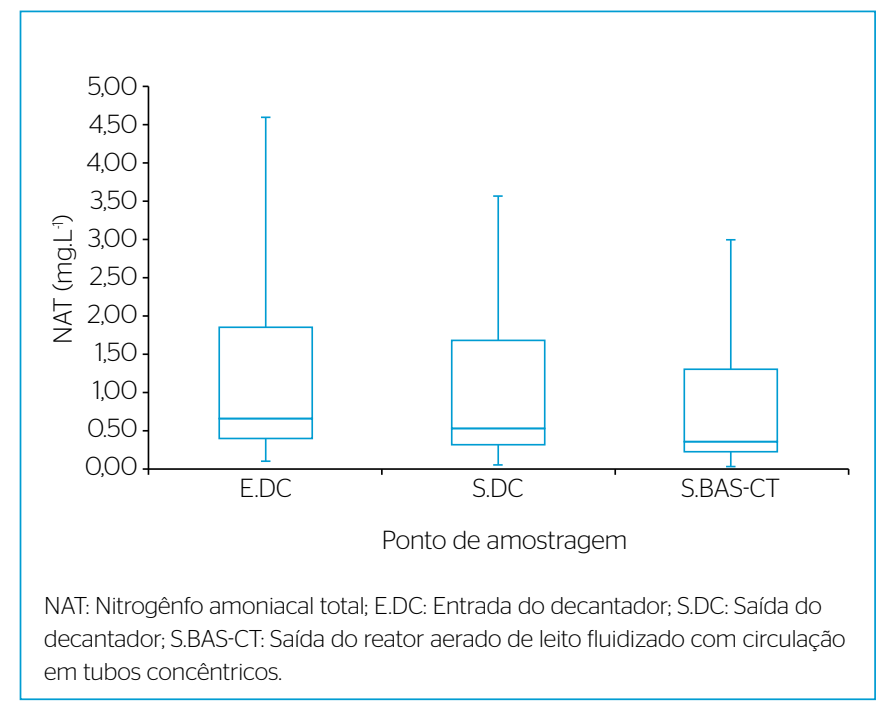

Figura 2 - Distribuição do NAT na entrada e na saída do decantador de coluna (E.DC e S.DC) e na saída do reator aerado de leito fluidizado com circulação em tubos concêntricos (S.BAS-CT). nas concentrações de DBO como ainda em DQO. Assim, tal fato pode estar afetando também as taxas de remoção de NAT (TIMMONS \& EBELING, 2010; ZHU \& CHEN, 2001).

Os valores da concentração de DBO durante o período avaliado no SRA são apresentados na Figura 3.

O valor médio calculado para a remoção de DBO no SRA foi de $48 \%$, similar ao obtido por Sánchez Ortiz e Matsumoto (2012), de 47\%, utilizando decantador convencional (DCV) seguido de BAS-CT e como meio de suporte o carvão ativado granulado. Davidson, Helwig e Summerfelt (2008), num SRA para truta-arco-íris, empregaram FSB com areia de $\mathrm{D}_{10}=0,11 \mathrm{~mm}$ e alcançaram resultados de remoção em DBO de 63\%; e com areias $\mathrm{D}_{10}=0,19 \mathrm{~mm}$, remoção de $\mathrm{DBO}$ de $60 \%$. Esses melhores resultados podem ter sido influenciados pela maior vazão de ar utilizada no reator, que foi de 7.200 L.h ${ }^{-1}$ durante o período de operação, possuindo maior capacidade para oxidar matéria orgânica. Remoções de 40 a $60 \%$ em DBO podem ser atingidas por meio de um reator biológico de membrana (MBR) aplicado em SRA marinhos, mas com TRH do efluente de até 9 h (VISVANATHAN; HUNG; JEGATHEESAN, 2008).

\section{Remoção da demanda química de oxigênio (DQO)}

Na Figura 4 podem ser observados os resultados das concentrações de DQO. Os valores indicam regularidade no comportamento, com maiores concentrações na E.DC e diminuição de DQO na S.DC e S.BAS-CT. Os valores máximos na concentração desse parâmetro devem-se ao decorrente desprendimento de material biológico contido no reator, por conta da geração e liberação de produtos solúveis no efluente e do envelhecimento e morte celular do biofilme (TIMMONS \& EBELING, 2010).

O valor da remoção média de DQO no SRA foi de 64,9\%, sendo inferior se comparado com os $77 \%$ de remoção obtidos por Sánchez

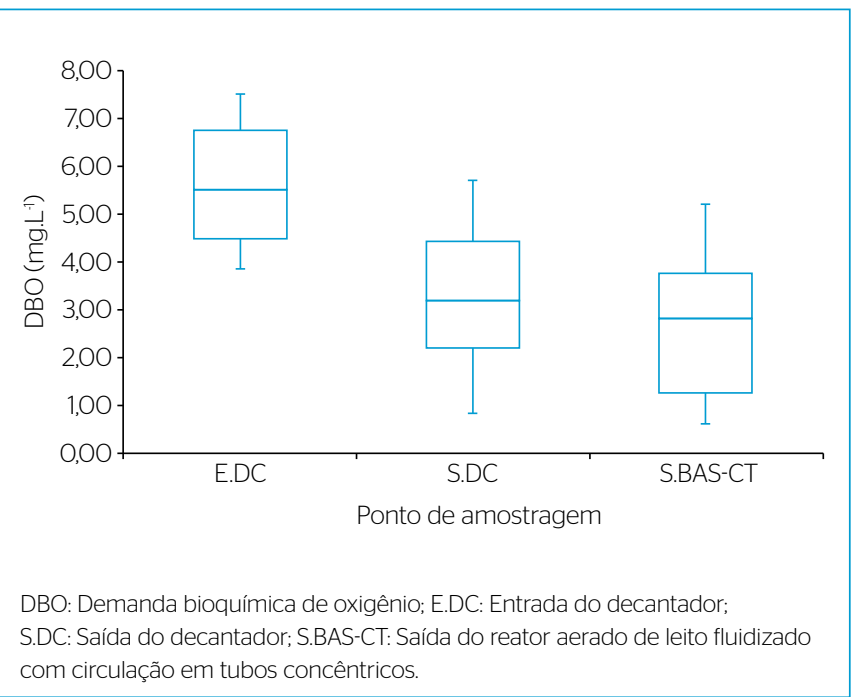

Figura 3 - Distribuição das concentrações de DBO na entrada e na saída do decantador de coluna (E.DC e S.DC) e na saída do reator aerado de leito fluidizado com circulação em tubos concêntricos (S.BAS-CT). 


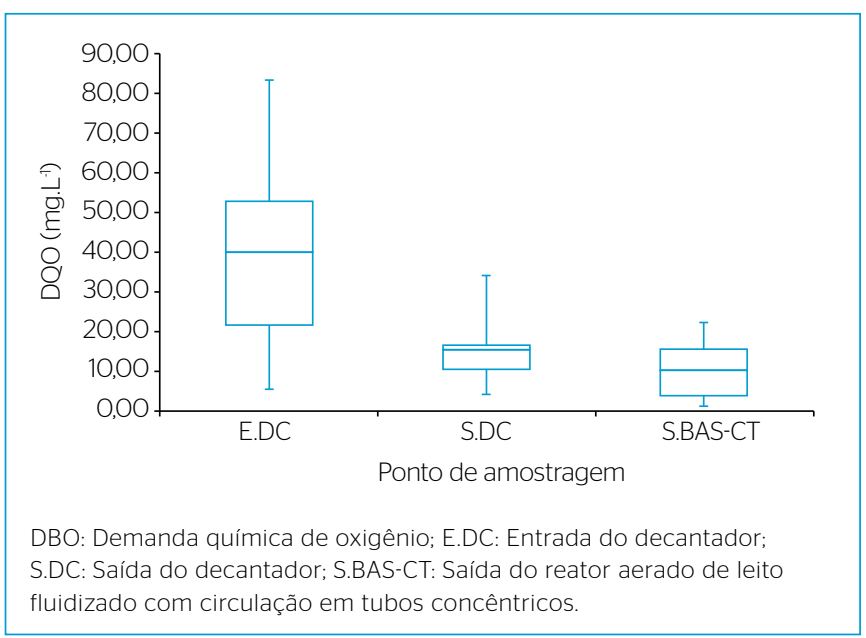

Figura 4 - Distnbuição das concentrações de DQO na entrada e na saída do decantador de coluna (E.DC. e S.DC) e na saída do reator aerado de leito fluidizado com circulação em cubos concêntricos (S. BAS-CT).

Ortiz e Matsumoto (2012) utilizando um sistema de tratamento de água similar. No início das operações foi necessária a adição de sal no SRA como medida profilática para a saúde dos peixes, para evitar a proliferação de doenças no sistema em função da possibilidade de queda na temperatura da água pelo começo do inverno, mas o sistema tinha aquecimento de água. A maior concentração de sal pode ser acompanhada pelo aumento de DQO do efluente tratado, diminuindo ligeiramente a remoção mediante um reator em batelada sequencial aeróbio, podendo levar até a inibição do processo biológico se a concentração do sal for muito elevada (DEORSOLA et al., 2013).

Constatou-se que nas datas em que foi adicionado sal no sistema houve diminuição na eficiência da remoção de DQO. Foi feita troca de água substituindo metade do volume de DC, em razão da presença de material sólido flutuante nas paredes da unidade, evitando assim a passagem desse material para o reator.

Segundo Visvanathan, Hung e Jegatheesan (2008), avaliando um reator de membrana com TRH de 2 até $9 \mathrm{~h}$, obteve-se remoção de DQO de 80 a 90\%. Os valores de remoção foram superiores aos obtidos na presente pesquisa, no entanto a tecnologia MBR é muito custosa se aplicada em sistemas com água salgada, podendo torná-la inviável no uso em SRA com água doce, além de mais TRH que essa tecnologia necessita para chegar a ditas porcentagens de remoção.

\section{CONCLUSÕES}

O DC e BAS-CT são unidades muito econômicas de construir por meio de materiais comercialmente disponíveis, assim como o meio de suporte utilizado, que tem alta área de superfície específica e baixo custo, assegurando a manutenção dos parâmetros ideais para o desenvolvimento da atividade aquícola em sistemas de reaproveitamento da água.

O SRA testado com sistema de tratamento de efluente com DC e BAS-CT mostrou ser adequado para remoção de NAT na manutenção da criação de tilápias, mantendo concentrações médias de $0,81 \mathrm{mg} . \mathrm{L}^{-1}$ de NAT no efluente final e remoções de $35,2 \%$, que garantem o normal funcionamento do sistema proposto.

A remoção de DBO foi de $48 \%$ e a concentração média na S.BAS-CT de 2,65 mg. $\mathrm{L}^{-1}$, o que não compromete a viabilidade da utilização do SRA no sistema de tratamento de efluente adotado.

A concentração de DQO foi reduzida em 64,9\% do efluente bruto, valor que permite a reutilização da água no SRA para cultivo de tilápias-do-nilo sem apresentar anormalidades na produção. A concentração média final encontrada no efluente foi de $10,1 \mathrm{mg} . \mathrm{L}^{-1}$.

\section{REFERÊNCIAS}

AMERICAN PUBLIC HEALTH ASSOCIATION - APHA. (2005). Standard methods for the examination of water and wastewater. 21. ed. Washington, D.C., Estados Unidos.

BRAZIL, B. L. (2006). Performance and operation of a rotating biological contactor in a tilapia recirculating aquaculture system. Aquacultural Engineering, v. 34, n. 3, p. 261-274.

BURUT-ARCHANAI, S.; EATON-RYE, J. J.; INCHAROENSAKDI, A.; POWTONGSOOK, S. (2013). Phosphorus removal in a closed recirculating aquaculture system using the cyanobacterium Synechocystis sp. PCC 6803 strain lacking the SphU regulator of the Pho regulon. Biochemical Engineering Journal, v. 74, p. $69-75$
DAVIDSON, J.; HELWIG, N.; SUMMERFELT, S. T. (2008). Fluidized sand biofilters used to remove ammonia, biochemical oxygen demand, total coliform bacteria, and suspended solids from an intensive aquaculture effluent. Aquacultural Engineering, v. 39, n. 1, p. 6-15.

DEORSOLA, A. B.; CAMARINHA, G. C.; CARVALHO, D. D.; SANT'ANNA JR., G. L. (2013). Biological treatment of saline wastewaters in an aerobic sequencing batch reactor. Environmental Progress \& Sustainable Energy, v. 32, n. 2, p. 198-205.

DOLAN, E.; MURPHY, N.; O'HEHIR, M. (2O13). Factors influencing optimal micro-screen drum filter selection for recirculating aquaculture systems. Aquacultural Engineering, v. 56, p. 42-50. 
GUTIERREZ-WING, M. T. \& MALONE, R. F. (2006). Biological filters in aquaculture: trends and research directions for freshwater and marine applications. Aquacultural Engineering, v. 34, n. 3 , p. 163-171.

JOHNSON, W. \& CHEN, S. (2006). Performance evaluation of radial/vertical flow clarification applied to recirculating aquaculture systems. Aquacultural Engineering, v. 34, n. 1, p. 47-55.

LAWSON, T. B. (1995). Fundamentals of aquacultural engineering. Nova York: Chapman \& Hall. 355 p.

LEKANG, O. (2013). Aquaculture Engineering. 2. ed. Oxford: WilleyBlackwell. 423 p.

MAIGUAL, Y.; SÁNCHEZ, I.; MATSUMOTO, T. (2013). Desempeño de tanques decantadores de sólidos en un sistema de recirculación para producción de tilapia. Revista MVZ Córdoba, v. 18, n. 2, p. $3.492-3.500$

MIRZOYAN, N.; TAL, Y.; GROSS, A. (2010). Anaerobic digestion of sludge from intensive recirculating aquaculture systems: review. Aquaculture, v. 306, n. 1-4, p. 1-6.

NICOLELLA, C.; VAN LOOSDRECHT, M. C. M.; HEIJNEN, J. J. (2000). Wastewater treatment with particulate biofilm reactors. Journal of Biotechnology, v. 80, n. 1, p. 1-33.

PFEIFFER, T. J.; OSBORN, A.; DAVIS, M. (2008). Particle sieve analysis for determining solids removal efficiency of water treatment components in a recirculating aquaculture system. Aquacultural Engineering, v. 39, n. 1, p. 24-29.

PIEDRAHITA, R. H. (2003). Reducing the potential environmental impact of tank aquaculture effluents through intensification and recirculation. Aquaculture, v. 226, n. 1-4, p. 35-44.
SÁNCHEZ ORTIZ, I. A. \& MATSUMOTO, T. (2012). Hydrodynamic characterization and performance evaluation of an aerobic three phase airlift fluidized bed reactor in a recirculation aquaculture system for Nile Tilapia production. Aquacultural Engineering, v. 47, p. 16-26.

SÁNCHEZ ORTIZ, I. A.; TEIXEIRA CORREIA, G.; SANTOS ARAÚJO, L.; GEBARA, D.; DALL'AGLIO SOBRINHO, M.; MATSUMOTO, T. (2010). Reactores aerobios de lecho fluidizado trifásico con circulación interna: caracterización hidrodinámica y del soporte. Revista Facultad de Ingenería Universidad de Antioquia, n. 56, p. 68-77.

SCHREIER, H. J.; MIRZOYAN, N.; SAITO, K. (2010). Microbial diversity of biological filters in recirculating aquaculture systems. Current Opinion in Biotechnology, v. 21, n. 3, p. 318-325.

SUMMERFELT, S. T. (2006). Design and management of conventional fluidized-sand biofilters. Aquacultural Engineering, v. 34, n. 3, p. 275-302

TIMMONS, M. B. \& EBELING, J. M. (2010). Recirculating aquaculture. 2. ed. Ítaca: Northeastern Regional Aquaculture Center. 948 p.

TIMMONS, M. B.; EBELING, J. M.; WHEATON, F.; SUMMERFELT, S. $\mathrm{VINCl}$, B. (2002). Sistemas de recirculación para la acuicultura. Chile: Fundación Chile. 747 p.

VAN DEN HENDE, S.; BEELEN, V.; BORE, G.; BOON, N.; VERVAEREN $H$. (2014). Up-scaling aquaculture wastewater treatment by microalgal bacterial flocs: from lab reactors to an outdoor raceway pond. Bioresource Technology, v. 159, p. 342-354.

VISVANATHAN, C; HUNG, N. Q; JEGATHEESAN, V. (2008). Hydrogenotrophic denitrification of synthetic aquaculture wastewater using membrane bioreactor. Process Biochemistry, v. 43, n. 6, p. 673-682

ZHU, S. \& CHEN, S. (2001). Effect of organic carbon on nitrification rate in fixed biofilm filters. Aquacultural Engineering, Essex, v. 25, p. 1-11. 